\title{
Identification and comparison of RCMV ALL 03 open reading frame (ORF) among several different strains of cytomegalovirus worldwide
}

\begin{abstract}
Background: Rat cytomegalovirus ALL-03 (Malaysian strain) which was isolated from a placenta and uterus of a house rat, Rattus rattus diardii has the ability to cross the placenta and infecting the fetus. To further elucidate the pathogenesis of the Malaysian strain of Rat Cytomegalovirus ALL-03 (RCMV ALL-03), detailed analysis on the viral genome sequence is crucial. Methods: Genome sequencing of RCMV ALL-03 was carried out in order to identify the open reading frame (ORF), homology comparison of ORF with other strains of CMV, phylogenetic analysis, classifying ORF with its corresponding conserved genes, and determination of functional proteins and grouping of gene families in order to obtain fundamental knowledge of the genome. Results: The present study revealed a total of 123 Coding DNA sequences (CDS) from RCMV ALL-03 with 37 conserved ORF domains as with all herpesvirus genomes. All the CDS possess similar function with RCMV-England followed by RCMV-Berlin, RCMV-Maastricht, and Human CMV. The phylogenetic analysis of RCMV ALL-03 based on conserving genes of herpes virus showed that the Malaysian RCMV isolate is closest to RCMV-English and RCMV-Berlin strains, with 99\% and 97\% homology, respectively. Similarly, it also demonstrated an evolutionary relationship between RCMV ALL-03 and other strains of herpesviruses from all the three subfamilies. Interestingly, betaherpesvirus subfamily, which has been shown to be more closely related with gammaherpesviruses as compared to alphaherpesviruses, shares some of the functional ORFs. In addition, the arrangement of gene blocks for RCMV ALL-03, which was conserved among herpesvirus family members was also observed in the RCMV ALL-03 genome. Conclusion: Genomic analysis of RCMV ALL-03 provided an overall picture of the whole genome organization and it served as a good platform for further understanding on the divergence in the family of Herpesviridae.
\end{abstract}

Keyword: Identification and comparison; Rat Cytomegalovirus ALL-03; RCMV ALL-03; Open reading frame; Cytomegalovirus 